\section{OPEN ACCESS}

Edited by:

Lauren M. Osborne,

Johns Hopkins University,

United States

Reviewed by:

Katherine Mcevoy,

Health Service Executive, Ireland

Miguel Meira E. Cruz,

Centro Cardiovascular da Faculdade

de Medicina da Universidade de

Lisboa, Portugal

*Correspondence:

Anna Wikman

anna.wikman@kbh.uu.se

Specialty section:

This article was submitted to

Women's Mental Health,

a section of the journal

Frontiers in Global Women's Health

Received: 02 November 2021

Accepted: 24 December 2021

Published: 31 January 2022

Citation:

Pitsillos T, Wikström A-K, Skalkidou A,

Derntl B, Hallschmid M, Lutz ND,

Ngai E, Sundström Poromaa I and

Wikman A (2022) Association

Between Objectively Assessed Sleep

and Depressive Symptoms During

Pregnancy and Post-partum.

Front. Glob. Womens Health

2:807817.

doi: $10.3389 / f g w h .2021 .807817$

\title{
Association Between Objectively Assessed Sleep and Depressive Symptoms During Pregnancy and Post-partum
}

\begin{abstract}
Tryfonas Pitsillos ${ }^{1}$, Anna-Karin Wikström ${ }^{1}$, Alkistis Skalkidou ${ }^{1}$, Birgit Derntt ${ }^{2,3}$, Manfred Hallschmid ${ }^{4,5,6}$, Nicolas D. Lutz ${ }^{4,7}$, Edith Ngai ${ }^{8,9}$, Inger Sundström Poromaa ${ }^{1}$ and Anna Wikman ${ }^{1 *}$

${ }^{1}$ Department of Women's and Children's Health, Uppsala University, Uppsala, Sweden, ${ }^{2}$ Department of Psychiatry and Psychotherapy, Tübingen Center for Mental Health, University of Tübingen, Tübingen, Germany, ${ }^{3}$ Lead Graduate School, University of Tübingen, Tübingen, Germany, ${ }^{4}$ Institute of Medical Psychology and Behavioral Neurobiology, University of Tübingen, Tübingen, Germany, ${ }^{5}$ German Center for Diabetes Research (DZD), Tübingen, Germany, ${ }^{6}$ Institute for Diabetes Research and Metabolic Diseases of the Helmholtz Center Munich at the University of Tübingen, Tübingen, Germany, ${ }^{7}$ Institute of Medical Psychology, Ludwig-Maximilians-Universität München, Munich, Germany, ${ }^{8}$ Department of Information Technology, Uppsala University, Uppsala, Sweden, ${ }^{9}$ Department of Electrical and Electronic Engineering, The University of Hong Kong, Hong Kong, Hong Kong SAR, China
\end{abstract}

Introduction: Sleep problems are common in pregnancy but many studies have relied only on self-reported sleep measures. We studied the association between objectively measured sleep and peripartum depressive symptoms in pregnant women.

Material and Methods: Sleep was assessed using Actiwatch accelerometers in a sample of 163 pregnant women in the late first (weeks 11-15) or early second trimester (weeks 16-19). Depressive symptoms were assessed in gestational weeks 17, 32 and at 6 weeks post-partum using the Edinburgh Postnatal Depression Scale (EPDS). Multiple linear regression and logistic regression analyses, adjusting for age, $\mathrm{BMI}$, pre-pregnancy smoking, ongoing mental health problems, trimester and season of sleep assessment were carried out to test the association between sleep and depression. Sleep was measured by total sleep time and sleep efficiency, whereas depression was indicated by depressive symptoms and depression caseness. Results are presented as unstandardized beta (B) coefficients or adjusted odds ratios (AOR) and 95\% confidence intervals $(\mathrm{Cl})$.

Results: Total sleep time ranged from 3 to $9 \mathrm{~h}$ (mean 7.1, SD 0.9) and average sleep efficiency was 83\% (SD 6.0). Women with the shortest total sleep time, i.e., in the lowest quartile $(<6.66 \mathrm{~h}$ ), reported higher depressive symptoms during pregnancy (week 17, $B$ $=2.13,95 \% \mathrm{Cl}$ 0.30-3.96; week 32, $B=1.70,95 \% \mathrm{Cl} 0.03-3.37$ ) but not post-partum. Their probability to screen positive for depression in gestational week 17 was increased more than 3-fold ( $\mathrm{AOR}=3.46,95 \% \mathrm{Cl} 1.07-11.51$ ) but unchanged with regards to gestational week 32 or 6 weeks post-partum. Sleep efficiency was not associated with depressive symptoms at any stage of pregnancy or post-partum.

Discussion: In one of the few studies to use objective sleep measures to date, mental health of pregnant women appeared to be affected by shortened sleep, with 
total sleep time being negatively associated with depressive symptoms in the early second and third trimester. This finding highlights the relevance of identifying and treating sleep impairments in pregnant women early during antenatal care to reduce the risk of concomitant depression.

Keywords: total sleep time, sleep efficiency, accelerometer, Edinburgh Postnatal Depression Scale, Sweden

\section{INTRODUCTION}

Pregnancy and the post-partum period are associated with an increased risk of developing mental health problems. The American Psychiatric Association's Diagnostic and Statistical Manual of Mental Disorders, fifth edition (DSM-5), specifies perinatal depression as a major depressive episode with onset during pregnancy or the first 4 weeks post-partum (1). However, as depression that begins later than 4 weeks after delivery or does not meet the full criteria for a major depressive episode may still cause harm and require treatment, experts in the field continue to define peripartum depression as a minor or major depressive episode occurring anytime during pregnancy or within the first year post-partum $(2,3)$. The condition affects $\sim 10$ to $15 \%$ of the global female population of childbearing age, with substantial variation across nations, partly explained by disparities in wealth inequality and maternal-child-health factors (4). Perinatal depression has major health and social impactsnot only for the becoming mother but also the offspring. Maternal suicidality and self-harm are crucial concerns among women who experience perinatal depression $(5,6)$. Furthermore, perinatal depression can lead to a variety of negative birth outcomes such as preterm birth, low birth weight, low APGAR score, and impaired breastfeeding (7-10). Perinatal depression also influences the mother's ability to parent and bond with the infant (11).

Multiple risk factors contributing to the development of perinatal depression have been identified, emphasizing the multidimensional nature of this condition. Socioeconomic factors such as maternal age, income, educational status, unemployment, smoking, domestic violence, an unsupportive partner, as well as a history of depression or other mental health conditions, hormonal factors and dysregulations of the HPA axis are possible determinants $(10,12-14)$. Across the majority of studies, a history of pre-pregnancy depression or mental health problems already at the start of pregnancy are typically the strongest risk factors for exacerbation of depression at any stage of pregnancy and the post-partum period $(15,16)$.

Pregnancy is commonly experienced to go along with increased sleep problems due to the tremendous anatomical, physiological and hormonal changes a pregnant woman's body undergoes (17-19). Typical self-reported pregnancy-related sleep problems include short sleep duration, poor sleep quality, sleep apnea, habitual snoring, and restless legs syndrome $(20,21)$. While many of these mostly subjective sleep problems are most prominent in the later stages of pregnancy-and may not necessarily be reflected by objective sleep measures relying on rigorous methodology [e.g., (22)] - many women complain of short sleep duration and poor sleep quality already in the first trimester (23-25). Sleep disturbances are also associated with depressive symptoms or clinical depression (26). In fact, impaired sleep is regarded as one of the most prominent symptoms in depressive patients. Notably, it is also a risk factor for developing depression, thus rendering sleep problems not just an epiphenomenon, but a predictor of depression (27). Common sleep disturbances related to depression are prolonged sleep latency, problems to maintain sleep, and early morning awakenings (26), thus implying a reduction of total sleep time. In fact, both short and long sleep duration has been suggested as an important factor in this context (28). Consequently, and in the context of pregnancy, sleep problems have been suggested to play a major role for the development of peripartum depression (29).

To date, there are few longitudinal studies demonstrating this relationship by using self-reported sleep problems during pregnancy as a predictor of later onset of depression. In one study four different sleep trajectories based on assessments at four time-points during pregnancy and up to 6 months postpartum were identified. The results indicated that women with the poorest sleep, with increasing severity of sleep problems during pregnancy, were more likely to develop depressive symptomatology in the post-partum period (30). Results from a large cohort study, collecting subjective sleep data during the second trimester of pregnancy, showed that poor sleep quality was positively correlated with more severe depressive symptoms during the second trimester and in the third month postpartum (31). One study evaluating the relationship between sleep quality in late pregnancy and recurrence of post-partum major depression through 28 weeks post-partum found poor sleep quality to predict recurrence after 4 weeks post-partum (32).

It should be pointed out that the majority of studies investigating sleep and perinatal depression have relied on subjective sleep assessments (using self-report questionnaires) and largely focused on sleep assessments in the third trimester and the post-partum period $(19,33,34)$. However, the use of self-report may be problematic due to discrepancies between subjective and objective sleep measurements $(22,35)$, especially in patients with depression, who tend to over-report sleep problems $(36,37)$. Presently, only one study has used actigraphy for collection of objective sleep data in early pregnancy to study the influence of sleep deficiency on stress and depressive symptoms. Their results showed that sleep loss in the first and early second trimester was strongly associated with stress and depressive symptoms in early pregnancy (38).

Given the paucity of longitudinal studies investigating the interaction of sleep and depression in the peripartum phase, the aim of this study was to extend the knowledge on sleep 
problems in pregnancy and their association with concomitant and later development of depression. Specifically, we collected objectively measured (i.e., accelerometry-derived) sleep data between gestational weeks 11-19 and examined the association between total sleep time and sleep efficiency and perinatal depressive symptoms across three time-points during pregnancy and the post-partum.

\section{MATERIALS AND METHODS \\ Study Population}

Data were obtained from (1) the Biology, Affect, Stress, Imaging and Cognition during pregnancy and post-partum (BASIC) study (39), and (2) the Safe Physical Activity in Pregnancy (SPAP) study, both carried out at the Department of Obstetrics and Gynecology, Uppsala University Hospital, Sweden.

Between September 2009 to November 2018 all pregnant, Swedish-speaking women of $\geq 18$ years of age without confidential personal data, who were scheduled for a routine ultrasound (between weeks 16 and 19) in Uppsala County, received a letter with an invitation to participate in the BASIC study at their first ultrasound appointment. Women who agreed to participate returned their written informed consent by post. Participants were asked to complete online surveys at gestational weeks 17 and 32, and at 6 weeks post-partum. Since April 22nd 2016, women attending either the first trimester ultrasound examination between gestational weeks 11-15 or the second trimester ultrasound examination between gestational weeks 16-19 have also been invited to participate in the SPAP study. Participation involves wearing an accelerometer for physical activity and sleep recordings without interruption for seven consecutive days. Inclusion criteria were: an uncomplicated pregnancy at recruitment, ability to use a wrist watch during work hours and age 18 years or older. Written informed consent was obtained from all participants at inclusion. Upon inclusion, brief demographic data were collected including ongoing chronic health disorders, medication, smoking, and height and weight.

Between April 22nd 2016 and September 18th 2018, 174 women participated in both the SPAP and BASIC studies, of whom 11 had insufficient sleep data and were excluded. Thus, 163 women were eligible for inclusion in the present study. Ethical approval was granted by the Regional Ethical Review Board in Uppsala, Sweden ([1] Dnr 2009/171, and [2] Dnr 2016/142).

\section{Measures}

\section{Participant Characteristics}

Information on participant characteristics was obtained from the standardized antenatal, obstetric and medical records and included age, parity (primiparous or multiparous), Body Mass Index (BMI) at first antenatal visit [categorized as $<25$ (underweight/normal weight) or $\geq 25$ (overweight/obese) $\left.\mathrm{kg} / \mathrm{m}^{2}\right]$, pre-pregnancy smoking (yes or no), trimester of sleep assessment (first or second), season of sleep assessment [November-February (winter) or March-October (not winter), based on seasonal differences in daylight hours]. Information on ongoing mental health problems was collected at the first antenatal visit and included self-reported ongoing depression, bipolar disorder, generalized anxiety disorder, panic disorder, attention deficit hyperactivity disorder, and personality disorder (categorized as presence of any ongoing mental disorder, yes or no).

\section{Sleep Recordings and Sleep Parameters}

Participants wore either an Actiwatch-2 or an Actiwatch Spectrum Plus accelerometer (Philips Respironics) on the nondominant wrist without interruption for seven consecutive days. The Actiwatch-2 is a lightweight, water-proof solid state piezo-electric accelerometer with a silicon photodiode whereas the Actiwatch Spectrum Plus has a MEMS type accelerometer and a color sensitive photodiode which detects light with wavelength range 400-900 nanometers. In line with standard actigraphy provisions, the accelerometer detects movements of the wrist between 0.5 and $2.0 \mathrm{G}$, with a frequency response range between 0.35 and $7.5 \mathrm{~Hz}$ and a sampling frequency of $32 \mathrm{~Hz}$. In comparison to polysomnography, which is the gold standard in sleep analysis, wearable sleep technology has been shown to be a reliable and validated method of assessing sleep characteristics $(40,41)$.

Participants were advised to stick to their everyday life during the study period. By pressing a button on the watch, participants recorded the time of going to bed in the evening and the time of waking up in the morning (to calculate time in bed). These events were used in the scoring algorithm to identify sleep periods, i.e., the interval between sleep onset and sleep offset. When the 7 days were completed, the accelerometer was returned by post and sleep data were extracted using Philips Actiware Software version 6.0.9. The amount of activity (number of movements in 30-s epochs and number of movements above a predefined threshold) was used for the determination of sleep and wake intervals. Individual actograms and sleep episodes were visually inspected before analyses to screen for adherence to the instructions, artifacts and malfunctioning. Participants were included in the analyses if they had a minimum of three valid sleep periods [the majority $(87.7 \%)$ had valid recordings for seven nights]. The raw data from the actograms were then transferred to and analyzed in MATLAB (version 9.4), obtaining numeric values of the sleep parameters for statistical analyses.

Two of the most commonly studied sleep parameters were included for analyses in the present study; total sleep time and sleep efficiency. Total sleep time (hours) was calculated by excluding the sum of periods of wakefulness during the sleep period. Sleep efficiency is the ratio (in percent) of total sleep time and time in bed and reflects time spent awake in bed, including both sleep onset latency and wake after sleep onset.

\section{Depressive Symptoms}

Depressive symptoms at gestational weeks 17 (i.e., around the time of actigraphy recording) and 32 (i.e., in the third trimester), and at 6 weeks post-partum were assessed with the Swedish version of the Edinburgh Postnatal Depression Scale, EPDS $(42,43)$. The EPDS is a scale including 10 items each scored from 0 to 3 (total score between 0 and 30 ). Higher scores indicate more severe symptoms. Scores of $\geq 13$ during pregnancy and $\geq 12$ post-partum on the EPDS suggest 
clinically relevant depressive symptoms (i.e., screen-positive), as previously validated in Swedish perinatal samples $(43,44)$. The EPDS has shown adequate reliability and validity (45).

\section{Statistical Analyses}

Descriptive statistics are reported as means and standard deviations (SD) or numbers ( $n$ ) and percentages (\%). Based on sample size and questions as to whether cut-offs from healthy populations are valid in pregnant samples, both sleep parameters were split in quartiles. To explore the relationship between total sleep time [defined as lowest quartile $(<6.66 \mathrm{~h})$ vs. the rest $(\geq 6.66 \mathrm{~h})$ ] and sleep efficiency [defined as lowest quartile ( $<80 \%$ sleep efficiency) vs. the rest ( $\geq 80 \%$ sleep efficiency)] and depressive symptoms at gestational weeks 17 and 32, and at 6 weeks post-partum, three multiple linear regression analyses were carried out. The models adjusted for confounding by age, BMI at first antenatal visit, pre-pregnancy smoking, any ongoing mental health problems at first antenatal visit, trimester of sleep assessment, and season of sleep assessment. Parity was assessed as a potential confounder but did not influence either the exposure or the outcome and was thus not included in the models. Results are presented as unstandardized regression coefficients (B) with 95\% confidence intervals (CI) including the $R^{2}$ value for each analysis. In the final models, assumptions of multiple regression were met including no evidence of collinearity; all variance inflation factors were $<2.0$, and unstandardized residuals were normally distributed. In addition, we also performed multiple logistic regression analyses on risk of being screen-positive for depression (EPDS score $\geq 13$ during pregnancy and $\geq 12$ post-partum) using the same adjustments as in the multiple linear regression analyses. Statistical analyses were performed using IBM SPSS Statistics version 27.0 (IBM Corp., Armonk, N.Y., USA).

\section{RESULTS}

Participant characteristics are shown in Table 1. On average, women were 31.8 (SD 4.6) years old at inclusion and just over half of women were multiparous $(n=85,52.1 \%)$. More than one-third was overweight or obese $(n=60,36.8 \%)$ at first antenatal visit, and $7.4 \%$ reported pre-pregnancy smoking $(n=12)$. Approximately $14 \%$ reported ongoing mental health problems at the first antenatal visit $(n=23)$. In a majority of women, sleep assessment was carried out in the second trimester ( $n=106,65.0 \%$ ), and in one-third of women during the winter months $(n=50,30.7 \%)$.

Total sleep time ranged from 3 to $9 \mathrm{~h}$ (mean 7.1, SD 0.9) and the average sleep efficiency was $83 \%$ (SD 6.0). Total sleep time and sleep efficiency were moderately and positively correlated $(r=0.48, p<0.001)$. EPDS scores were available for 146 (89.6\%) participants at gestational week 17, 148 (90.8\%) women at gestational week 32, and $137(84.0 \%)$ women at 6 weeks postpartum. Mean EPDS scores were 6.0 (SD 5.0), 5.7 (SD 4.7) and 5.6 (SD 4.6) at gestational week 17, 32 and at 6 weeks post-partum, respectively. The prevalence of clinically relevant depressive symptoms (i.e., screen-positive women) was $12.9 \%$ ( $n$
TABLE 1 | Participant characteristics $(n=163)$.

\begin{tabular}{lc}
\hline Characteristic & Mean (SD) or $\boldsymbol{n}$ (\%) \\
\hline Age, years & $31.8(4.6)$ \\
Parity & \\
$\quad$ Primiparous & $73(44.8)$ \\
$\quad$ Multiparous & $85(52.1)$ \\
BMl (kg/m²), first antenatal visit & $24.7(4.7)$ \\
$<25$ (underweight/normal weight) & $100(61.3)$ \\
$\geq 25$ (overweight/obese) & $60(36.8)$ \\
Pre-pregnancy smoking & $12(7.4)$ \\
Yes & $151(92.6)$ \\
No & \\
Ongoing mental health problems, first antenatal visit* & \\
Yes & $23(14.1)$ \\
No & $139(85.3)$ \\
Trimester of sleep assessment & \\
First & $57(35.0)$ \\
Second & $106(65.0)$ \\
Winter & \\
\hline Not winter & $50(30.7)$ \\
\hline
\end{tabular}

BMI, Body Mass Index, at first antenatal visit; $n$, number of participants; SD, standard deviation.

Missing cases for parity $n=5$ (3.1\%), BMI $n=3(1.9 \%)$, ongoing mental health problems $n=1(0.6 \%)$.

*Including self-reported ongoing depression, bipolar disorder, generalized anxiety disorder, panic disorder, attention deficit hyperactivity disorder, and personality disorder.

$=21)$ at gestational week $17,8.6 \%(n=14)$ at week 32 , and $9.2 \%$ $(n=15)$ at 6 weeks post-partum.

Table 2 shows the results from the regression analyses. In gestational week 17, women with total sleep time in the lowest quartile reported significantly more pronounced depressive symptoms (adjusted for age, BMI at first antenatal visit, prepregnancy smoking, ongoing mental health problems, trimester of sleep assessment, and season of sleep assessment; $B=$ 2.13, 95\% CI 0.30-3.96) than women with total sleep time in the remaining quartiles. Sleep efficiency was unrelated to depressive symptoms at this time-point. In the adjusted model, older age was associated with fewer depressive symptoms ( $B$ $=-0.22,95 \% \mathrm{CI}-0.40$ to -0.04$)$, whereas ongoing mental health problems at first antenatal visit were associated with more severe depressive symptoms ( $B=3.74$, 95\% CI 1.525.97). In addition, women who completed sleep assessment during the winter months (November-February) reported more severe depressive symptoms ( $B=2.01,95 \%$ CI $0.23-3.80)$. The model explained $24 \%$ of the variance in depressive symptoms in gestational week 17. Table 3 shows the sleep variables in relation to the number of women who were screen-positive for depression at the different time-points. Women who had a total sleep time in the lowest quartile were more than three times more likely to be screen-positive for depression at this stage of pregnancy $(\mathrm{OR}=3.46$, 95\% CI $1.07-$ 11.51). Here, again, sleep efficiency was not associated with depressive symptoms. 
TABLE 2 | Multiple linear regression analyses of the association between objectively assessed sleep parameters and depressive symptoms on the EPDS at gestational weeks 17, 32 and 6 weeks post-partum adjusting for age, BMl at first antenatal visit, pre-pregnancy smoking, ongoing mental health problems at first antenatal visit, trimester at sleep assessment and season at sleep assessment.

\begin{tabular}{|c|c|c|c|c|c|c|}
\hline \multirow[b]{2}{*}{ Characteristics } & \multicolumn{2}{|c|}{$\begin{array}{c}\text { Depressive symptoms gestational } \\
\text { week } 17\end{array}$} & \multicolumn{2}{|c|}{$\begin{array}{c}\text { Depressive symptoms gestational } \\
\text { week } 32\end{array}$} & \multicolumn{2}{|c|}{$\begin{array}{l}\text { Depressive symptoms } \\
\text { post-partum week six }\end{array}$} \\
\hline & $\begin{array}{l}\text { Unstandardized } \\
\text { regression } \\
\text { coefficients, } \\
\text { B }(95 \% \mathrm{Cl})\end{array}$ & $p$ & $\begin{array}{l}\text { Unstandardized } \\
\text { regression } \\
\text { coefficients, } \\
\text { B }(95 \% \mathrm{Cl})\end{array}$ & $p$ & $\begin{array}{l}\text { Unstandardized } \\
\text { regression } \\
\text { coefficients, } \\
\text { B }(95 \% \mathrm{Cl})\end{array}$ & $p$ \\
\hline Total Sleep Time (lowest quartile vs. the rest) & $2.13(0.30-3.96)$ & 0.03 & $1.70(0.03-3.37)$ & 0.05 & $-0.63(-2.37$ to 1.12$)$ & 0.48 \\
\hline Sleep Efficiency (lowest quartile vs. the rest) & $-0.48(-2.47$ to 1.52$)$ & 0.93 & $-1.40(-3.28$ to 0.49$)$ & 0.15 & $-1.09(-3.09$ to 0.91$)$ & 0.28 \\
\hline Age & $-0.22(-0.40$ to -0.04$)$ & 0.02 & $-0.07(-0.23$ to 0.10$)$ & 0.43 & $-0.05(-0.22$ to 0.12$)$ & 0.54 \\
\hline BMI (overweight/obese vs. normal weight) & $1.14(-0.45$ to 2.73$)$ & 0.16 & $1.64(0.21-3.06)$ & 0.03 & $0.78(-0.72$ to 2.29$)$ & 0.31 \\
\hline Pre-pregnancy smoking (yes vs. no) & $2.55(-0.51$ to 5.60$)$ & 0.11 & $2.51(-0.16$ to 5.18$)$ & 0.07 & $3.56(0.86-6.25)$ & 0.01 \\
\hline Ongoing mental health problems (yes vs. no) & $3.74(1.52-5.97)$ & 0.001 & $5.50(3.52-7.49)$ & $<0.001$ & $4.72(2.75-6.68)$ & $<0.001$ \\
\hline Trimester of sleep assessment (first vs. second) & $0.58(-1.24$ to 2.40$)$ & 0.55 & $1.18(-0.63$ to 2.98$)$ & 0.20 & $0.32(-1.62$ to 2.27$)$ & 0.74 \\
\hline Season of sleep assessment (winter vs. not winter) & $2.01(0.23-3.80)$ & 0.03 & $1.81(0.06-3.55)$ & 0.05 & $1.67(-0.17$ to 3.51$)$ & 0.08 \\
\hline$R^{2}$ & 0.24 & & 0.31 & & 0.25 & \\
\hline
\end{tabular}

Cl, confidence interval; EPDS, Edinburgh Postnatal Depression Scale.

Statistically significant coefficients $(p<0.05)$ are marked in bold.

TABLE 3 | Multiple logistic regression analyses of the association between objectively assessed sleep parameters and being screen-positive for ongoing depression on the EPDS in gestational weeks 17, 32 and 6 weeks post-partum.

\begin{tabular}{|c|c|c|c|c|c|c|}
\hline \multirow[b]{2}{*}{ Characteristics } & \multicolumn{2}{|c|}{$\begin{array}{c}\text { Screen-positive for depression } \\
\text { gestational week } 17\end{array}$} & \multicolumn{2}{|c|}{$\begin{array}{c}\text { Screen-positive for depression } \\
\text { gestational week } 32\end{array}$} & \multicolumn{2}{|c|}{$\begin{array}{l}\text { Screen-positive for depression } \\
\text { post-partum week six }\end{array}$} \\
\hline & AOR (95\% Cl) & $p$ & AOR (95\% Cl) & $p$ & AOR (95\% Cl) & $p$ \\
\hline Total Sleep Time (lowest quartile vs. the rest) & $3.46(1.07-11.51)$ & 0.04 & $1.18(0.25-5.55)$ & 0.84 & $0.66(0.15-2.98)$ & 0.59 \\
\hline Sleep Efficiency (lowest quartile vs. the rest) & $0.75(0.21-3.08)$ & 0.76 & $0.32(0.03-3.16)$ & 0.33 & $0.25(0.03-2.45)$ & 0.23 \\
\hline
\end{tabular}

Cl, confidence interval; EPDS, Edinburgh Postnatal Depression Scale.

Statistically significant adjusted odds ratios, AOR ( $p<0.05)$, are marked in bold.

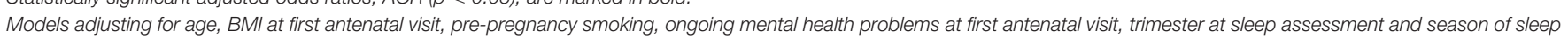
assessment (winter vs. not winter).

In gestational week 32, adjusting for all other variables in the model, total sleep time in the lowest quartile $(B=1.70,95 \%$ CI $0.03-3.37)$, overweight or obese BMI ( $B=1.64,95 \%$ CI 0.21 3.06), ongoing mental health problems at first antenatal visit $(B=5.51,95 \%$ CI $3.52-7.49)$ and sleep assessment during the winter months $(B=1.81,95 \%$ CI $0.06-3.55)$ were associated with depressive symptoms, whereas low sleep efficiency had no influence on depressive symptoms at week 32 (Table 2). The model explained $31 \%$ of variance in depressive symptoms in gestational week 32. However, none of the sleep variables influenced the risk of being screen-positive for depression in gestational week 32 (Table 3 ).

None of the sleep variables were associated with depressive symptoms at 6 weeks post-partum (Tables 2, 3). Instead, depressive symptoms in post-partum week six were predicted by ongoing mental health problems at the first antenatal visit $(B=4.72,95 \%$ CI $2.75-6.68)$ and pre-pregnancy smoking $(B=3.56,95 \%$ CI $0.86-6.25)$, after adjustment for all other variables in the model. The model in post-partum week six explained $25 \%$ of variance in depressive symptoms.

\section{DISCUSSION}

The main finding of this study was that pregnant women with sleep duration in the lowest quartile reported higher levels of depressive symptoms during pregnancy. This relationship was not only manifested at the time of the sleep recording, i.e., where it is unclear if the decreased sleep duration may be a symptom of ongoing depression or vice versa, but also later, in the third trimester of pregnancy, suggesting that short sleep durations may be a risk factor for developing depression. These findings were also independent of the women's age, BMI at first antenatal visit, smoking history, and the ongoing mental health problems some women reported at the first antenatal visit. Further, short sleep duration in the first or early second trimester of pregnancy was associated with increased risk of being screen-positive for depression in gestational week 17, but not at any later stage in pregnancy or post-partum. Finally, short sleep duration or poor sleep efficiency were not associated with depressive symptoms after childbirth.

Based on objective measurements, the findings of this study confirm prior findings of women's subjective reporting 
of sleep disturbances and their risk of developing depressive symptoms during pregnancy $(30,31,33)$. Thus, this study has added important value to the subjective reports of women by establishing that poor sleep, in this case objectively evaluated by actigraphy, may contribute to an increased risk of depression during pregnancy. Women who had a total sleep time of $<6.66 \mathrm{~h}$ before gestational week 20 reported on average 2.1 scale steps higher on the EPDS and were more than three times more likely to be screen-positive for depression in gestational week 17. This finding suggests that pregnant women who complain of short sleep duration in antenatal care should be further assessed for potential concomitant depression. We also found that women with sleep time $<6.66 \mathrm{~h}$ reported on average 1.7 scale steps higher on the EPDS in gestational week 32, suggesting that sleep problems may maintain and prolong existing depressive symptoms or lead to development of depressive symptoms during pregnancy. The latter finding is also in line with metaanalytical approaches to disentangle the directional relationship between sleep disturbances and depression, where evidence at hand suggest that sleep problems, especially those reflecting wakefulness, precede the onset of depression. In contrast, little support has been found for a predictive role of depressive symptoms in the development of sleep disturbances (46). There were no association between total sleep time and being screenpositive for depression in gestational week 32, which may be explained by a lack of power to detect this association.

Although it could have been anticipated that sleep efficiency, which incorporates both sleep onset latency and wake after sleep onset (both of which are greatly affected in insomnia), would be an independent predictor of depressive symptoms in pregnancy, we did not find any relationship between objectively assessed sleep efficiency and depressive symptoms. Despite total sleep time and sleep efficiency being moderately and positively correlated shorter sleep durations for several consecutive nights may not necessarily imply poorer sleep efficiency, on the contrary, it might even increase sleep efficiency due to being constantly overtired. Multiple awakenings are common in the beginning of pregnancy, due to normal pregnancy-related causes such as nycturia, nausea, vomiting or pain, and hence, the association with depressive symptoms may be weakened (23-25). This study did not have the power to characterize sleep disturbances in pregnant women with depression, but future studies should aim to objectively describe the sleep problems that depressed women may suffer from to enhance our understanding of how to best help affected women.

Results of the present study were not in line with findings suggesting that poor sleep in pregnancy is associated with postpartum depression (33). This may be explained by the fact that studies on post-partum depression have carried out their sleep assessments in late pregnancy or post-partum, whereas women participating in the present study recorded their sleep patterns during the first half of pregnancy. These findings also emphasize that post-partum depression, in contrast to antenatal depression, likely comes with its own set of risk factors, where partner support, delivery complications, infant temperament and hormonal factors might play a more pivotal role (10). Further, sleep disturbances in the first half of pregnancy can have different causes than sleep disturbances in the post-partum period, meaning that some women who have unaffected sleep during pregnancy may very well-develop sleep disturbances after they have given birth.

While this study has added some information on the relationship between short sleep duration and depressive symptoms in pregnancy, it does not come without limitations. The major drawback of the study was the relatively low number of women who were screen-positive for depression. As recruitment targeted women scheduled for routine ultrasound assessment in pregnancy, the majority were healthy and at low risk of depression. This is reflected in the overall low mean EPDS scores at each time point, and in the relatively low proportion of women with ongoing mental health problems at the first antenatal visit. For this reason, we have limited power to answer some highly relevant questions like the impact on clinically relevant depression, and sleep characteristics in women with depression. Of course, it must be noted also that perinatal depression was assessed using self-report (i.e., the EPDS), and not by clinical interview. Further, as women had missing EPDS scores from some of the time-points, we refrained from evaluating how sleep impacts perinatal depression trajectories. Although many women were invited to participate we lack information on the response rate for the SPAP study. Thus, the study did not include a population-based sample, which may influence the generalizability of results. Further, it has recently been suggested that the interaction between insomnia, sleep apnea and circadian misalignment contribute to explaining the relationship between sleep deprivation and depression in pregnancy (47). However, as we lack information on some of these important aspects, we were unable to explore these factors as contributors to reduced total sleep in the present study. This, however, warrants further research in this population. Despite these limitations it is important to highlight that the major strength of the study is the objectively assessed sleep parameters.

\section{CONCLUSION}

Based on objective measurements of sleep, the findings of this study confirm prior findings of women's subjective reporting of poor sleep and their risk of developing depressive symptoms during pregnancy. Although short sleep duration or poor sleep efficiency were not associated with depressive symptoms postpartum, many of the pregnant women included were affected by objectively measured poor sleep, in terms of shorter sleep duration associated with depressive symptoms in the early second and third trimester. These results point to the importance of addressing sleep impairments in pregnant women early during antenatal care which may attenuate the risk of concomitant depressive symptoms.

\section{DATA AVAILABILITY STATEMENT}

The raw data supporting the conclusions of this article will be made available by the authors, without undue reservation, upon reasonable request and with necessary ethics approval. 


\section{ETHICS STATEMENT}

The studies involving human participants were reviewed and approved by the Regional Ethical Review Board in Uppsala, Sweden (Dnr 2009/171, and Dnr 2016/142). The participants provided their written informed consent to participate in this study.

\section{AUTHOR CONTRIBUTIONS}

AS, IS, and A-KW: conceptualization and design. MH, NL, EN, TP, IS, and AW: data acquisition and analysis. AW

\section{REFERENCES}

1. American Psychiatric Association. Diagnostic and Statistical Manual of Mental Disorders. 5th ed. DSM-5. Arlington, VA: American Psychiatric Publishing (2013).

2. Wisner KL, Moses-Kolko EL, Sit DKY. Postpartum depression: a disorder in search of a definition. Arch Womens Ment Health. (2010) 13:37-40. doi: 10.1007/s00737-009-0119-9

3. Gaynes BN, Gavin N, Meltzer-Brody S, Lohr KN, Swinson T, Gartlehner G, et al. Perinatal depression: prevalence, screening accuracy, and screening outcomes. Evid Rep Technol Assess. (2005) 119:1-8. doi: 10.1037/e439372005-001

4. Hahn-Holbrook J, Cornwell-Hinrichs T, Anaya I. Economic and health predictors of national postpartum depression prevalence: a systematic review, meta-analysis, and meta-regression of 291 studies from 56 Countries. Front Psychiatry. (2017) 8:248. doi: 10.3389/fpsyt.2017.00248

5. Esscher A, Essén B, Innala E, Papadopoulos FC, Skalkidou A, SundströmPoromaa I, et al. Suicides during pregnancy and 1 year postpartum in Sweden, 1980-2007. Br J Psychiatry. (2016) 208:462-9. doi: 10.1192/bjp.bp. 114.161711

6. Nayak AS, Nachane HB. Maternal anthropometric determinants as risk markers of suicidality and severity of illness in women with postnatal depression. J Postgrad Med. (2020) 66:11-6. doi: 10.4103/jpgm.JPGM_541_18

7. Dowse E, Chan S, Ebert L, Wynne O, Thomas S, Jones D, et al. Impact of perinatal depression and anxiety on birth outcomes: a retrospective data analysis. Matern Child Health J. (2020) 24:718-26. doi: 10.1007/s10995-020-02906-6

8. Eastwood J, Ogbo FA, Hendry A, Noble J, Page A, Early Years Research Group (EYRG). The Impact of Antenatal Depression on Perinatal Outcomes in Australian Women. PLoS ONE. (2017) 12:e0169907. doi: 10.1371/journal.pone.0169907

9. Grigoriadis S, VonderPorten EH, Mamisashvili L, Tomlinson G, Dennis C$\mathrm{L}$, Koren $\mathrm{G}$, et al. The impact of maternal depression during pregnancy on perinatal outcomes: a systematic review and meta-analysis. J Clin Psychiatry. (2013) 74:e321-41. doi: 10.4088/JCP.12r07968

10. Wikman A, Axfors C, Iliadis SI, Cox J, Fransson E, Skalkidou A. Characteristics of women with different perinatal depression trajectories. $J$ Neurosci Res. (2020) 98:1268-82. doi: 10.1002/jnr.24390

11. Erickson N, Julian M, Muzik M. Perinatal depression, PTSD, and trauma: impact on mother-infant attachment and interventions to mitigate the transmission of risk. Int Rev Psychiatry Abingdon Engl. (2019) 31:245-63. doi: 10.1080/09540261.2018.1563529

12. ACOG Committee Opinion No. 757: screening for perinatal depression. Obstet Gynecol. (2018) 132:e208-12. doi: 10.1097/AOG.0000000000002927

13. Meltzer-Brody S. New insights into perinatal depression: pathogenesis and treatment during pregnancy and postpartum. Dialogues Clin Neurosci. (2011) 13:89-100. doi: 10.31887/DCNS.2011.13.1/smbrody

14. O'Hara MW, McCabe JE. Postpartum depression: current status and future directions. Annu Rev Clin Psychol. (2013) 9:379-407. doi: 10.1146/annurev-clinpsy-050212-185612 and TP: drafting the work. All authors interpretation of data, revising the work critically for important intellectual content, and authors agreed to be accountable for all aspects of the work and approved the final version to be published.

\section{FUNDING}

IS was supported by a grant from the Swedish Research Council 2020-01801. MH was supported by the German Federal Ministry of Education and Research (BMBF) to the German Center for Diabetes Research (DZD e.V.; 01GI0925).

15. Munk-Olsen T, Laursen TM, Mendelson T, Pedersen CB, Mors O, Mortensen PB. Risks and predictors of readmission for a mental disorder during the postpartum period. Arch Gen Psychiatry. (2009) 66:189. doi: 10.1001/archgenpsychiatry.2008.528

16. Silverman ME, Reichenberg A, Savitz DA, Cnattingius S, Lichtenstein P, Hultman CM, et al. The risk factors for postpartum depression: a populationbased study. Depress Anxiety. (2017) 34:178-87. doi: 10.1002/da.22597

17. Nowakowski S, Meers J, Heimbach E. Sleep and women's health. Sleep Med Res. (2013) 4:1-22. doi: 10.17241/smr.2013.4.1.1

18. Sahota PK, Jain SS, Dhand R. Sleep disorders in pregnancy. Curr Opin Pulm Med. (2003) 9:477-83. doi: 10.1097/00063198-200311000-00005

19. Sedov ID, Cameron EE, Madigan S, Tomfohr-Madsen LM. Sleep quality during pregnancy: a meta-analysis. Sleep Med Rev. (2018) 38:168-76. doi: 10.1016/j.smrv.2017.06.005

20. Lau Y, Cheng LJ, Chee DGH, Zhao M, Wong SH, Wong SN, et al. High body mass index and sleep problems during pregnancy: a meta-analysis and meta-regression of observational studies. J Sleep Res. (2021) e13443. doi: 10.1111/jsr.13443. [Epub ahead of print].

21. Wesström J, Skalkidou A, Manconi M, Fulda S, Sundström-Poromaa I. Pre-pregnancy Restless Legs Syndrome (Willis-Ekbom Disease) is associated with perinatal depression. J Clin Sleep Med. (2014) 10:527-33. doi: 10.5664/jcsm.3704. [Epub ahead of print].

22. Zinke K, Lehnert V, Fritsche A, Preissl H, Hallschmid M. Pregnant women do not display impaired memory formation across one night of sleep. J Sleep Res. (2021) 30:e13204. doi: 10.1111/jsr.13204

23. Ertmann RK, Nicolaisdottir DR, Kragstrup J, Siersma V, Lutterodt MC. Sleep complaints in early pregnancy. A cross-sectional study among women attending prenatal care in general practice. BMC Pregnancy Childbirth. (2020) 20:123. doi: 10.1186/s12884-020-2813-6

24. Tsai S-Y, Lee P-L, Lin J-W, Lee C-N. Cross-sectional and longitudinal associations between sleep and health-related quality of life in pregnant women: a prospective observational study. Int J Nurs Stud. (2016) 56:45-53. doi: 10.1016/j.ijnurstu.2016.01.001

25. Wilson DL, Barnes M, Ellett L, Permezel M, Jackson M, Crowe SF. Decreased sleep efficiency, increased wake after sleep onset and increased cortical arousals in late pregnancy: decreased sleep efficiency in late pregnancy. Aust $N$ Z J Obstet Gynaecol. (2011) 51:38-46. doi: 10.1111/j.1479-828X.2010.01252.x

26. Riemann D, Krone LB, Wulff K, Nissen C. Sleep, insomnia, and depression. Neuropsychopharmacol. (2020) 45:74-89. doi: 10.1038/s41386-019-0411-y

27. Fang H, Tu S, Sheng J, Shao A. Depression in sleep disturbance: a review on a bidirectional relationship, mechanisms and treatment. J Cell Mol Med. (2019) 23:2324-32. doi: 10.1111/jcmm.14170

28. Zhai L, Zhang H, Zhang D. Sleep duration and depression among adults: a meta-analysis of prospective studies. Depress Anxiety. (2015) 32:664-70. doi: $10.1002 /$ da. 22386

29. Okun ML. Disturbed sleep and postpartum depression. Curr Psychiatry Rep. (2016) 18:66. doi: 10.1007/s11920-016-0705-2

30. Tomfohr LM, Buliga E, Letourneau NL, Campbell TS, Giesbrecht GF. Trajectories of sleep quality and associations with mood during the perinatal period. Sleep. (2015) 38:1237-45. doi: 10.5665/sleep.4900 
31. Gao M, Hu J, Yang L, Ding N, Wei X, Li L, et al. Association of sleep quality during pregnancy with stress and depression: a prospective birth cohort study in China. BMC Pregnancy Childbirth. (2019) 19:444. doi: 10.1186/s12884-019-2583-1

32. Okun ML, Hanusa BH, Hall M, Wisner KL. Sleep complaints in late pregnancy and the recurrence of postpartum depression. Behav Sleep Med. (2009) 7:10617. doi: 10.1080/15402000902762394

33. Maghami M, Shariatpanahi SP, Habibi D, Heidari-Beni M, Badihian N, Hosseini $\mathrm{M}$, et al. Sleep disorders during pregnancy and postpartum depression: a systematic review and meta-analysis. Int J Dev Neurosci. (2021) 81:469-78. doi: 10.1002/jdn.10118

34. McEvoy KM, Rayapati D, Washington Cole KO, Erdly C, Payne JL, Osborne LM. Poor postpartum sleep quality predicts subsequent postpartum depressive symptoms in a high-risk sample. J Clin Sleep Med. (2019) 15:130310. doi: $10.5664 /$ jcsm.7924

35. Baker FC, Maloney S, Driver HS. A comparison of subjective estimates of sleep with objective polysomnographic data in healthy men and women. $J$ Psychosom Res. (1999) 47:335-41. doi: 10.1016/S0022-3999(99)00017-3

36. Rotenberg VS, Indursky $P$, Kayumov L, Sirota P, Melamed Y. The relationship between subjective sleep estimation and objective sleep variables in depressed patients. Int J Psychophysiol. (2000) 37:291-7. doi: 10.1016/S0167-8760(00)00110-0

37. Van Ravesteyn LM, Tulen JHM, Kamperman AM, Raats ME, Schneider AJ, Birnie E, et al. Perceived sleep quality is worse than objective parameters of sleep in pregnant women with a mental disorder. J Clin Sleep Med. (2014) 10:1137-41. doi: 10.5664/jcsm.4118

38. Okun ML, Kline CE, Roberts JM, Wettlaufer B, Glover K, Hall M. Prevalence of sleep deficiency in early gestation and its associations with stress and depressive symptoms. J Womens Health. (2013) 22:1028-37. doi: 10.1089/jwh.2013.4331

39. Axfors C, Bränn E, Henriksson HE, Hellgren C, Kunovac Kallak T, Fransson E, et al. Cohort profile: the Biology, Affect, Stress, Imaging and Cognition (BASIC) study on perinatal depression in a population-based Swedish cohort. BMJ Open. (2019) 9:e031514. doi: 10.1136/bmjopen-2019-031514

40. Marino M, Li Y, Rueschman MN, Winkelman JW, Ellenbogen JM, Solet JM, et al. Measuring sleep: accuracy, sensitivity, and specificity of wrist actigraphy compared to polysomnography. Sleep. (2013) 36:1747-55. doi: $10.5665 /$ sleep. 3142

41. Sadeh A, Tikotzky L, Scher A. Parenting and infant sleep. Sleep Med Rev. (2010) 14:89-96. doi: 10.1016/j.smrv.2009.05.003

42. Cox JL, Holden JM, Sagovsky R. Detection of postnatal depression: development of the 10-item Edinburgh Postnatal Depression Scale. $\mathrm{Br} J$ Psychiatry. (1987) 150:782-6. doi: 10.1192/bjp.150.6.782
43. Wickberg B, Hwang CP. The Edinburgh Postnatal Depression Scale: validation on a Swedish community sample. Acta Psychiatr Scand. (1996) 94:181-4. doi: 10.1111/j.1600-0447.1996. tb09845.x

44. Rubertsson C, Börjesson K, Berglund A, Josefsson A, Sydsjö G. The Swedish validation of Edinburgh Postnatal Depression Scale (EPDS) during pregnancy. Nord J Psychiatry. (2011) 65:414-8. doi: 10.3109/08039488.2011.590606

45. Affonso DD, De AK, Horowitz JA, Mayberry LJ. An international study exploring levels of postpartum depressive symptomatology. J Psychosom Res. (2000) 49:207-16. doi: 10.1016/S0022-3999(00)00176-8

46. Lovato N, Gradisar M. A meta-analysis and model of the relationship between sleep and depression in adolescents: recommendations for future research and clinical practice. Sleep Med Rev. (2014) 18:521-9. doi: 10.1016/j.smrv.2014.03.006

47. Meira e Cruz M, Sweetman, A. Insomnia, sleep apnea, and circadian misalignment as a "three-arm" contributor to anxiety and depression during pregnancy. Sleep Vigilance. (2021) 5:333-5. doi: 10.1007/s41782-021-0 0163-3

Conflict of Interest: Over the past five years, IS has served occasionally on advisory boards or acted as invited speaker at scientific meetings for Asarina Pharma, Bayer Health Care, Gedeon Richter, Peptonics, Shire/Takeda, and Sandoz.

The remaining authors declare that the research was conducted in the absence of any commercial or financial relationships that could be construed as a potential conflict of interest.

Publisher's Note: All claims expressed in this article are solely those of the authors and do not necessarily represent those of their affiliated organizations, or those of the publisher, the editors and the reviewers. Any product that may be evaluated in this article, or claim that may be made by its manufacturer, is not guaranteed or endorsed by the publisher.

Copyright (c) 2022 Pitsillos, Wikström, Skalkidou, Derntl, Hallschmid, Lutz, Ngai, Sundström Poromaa and Wikman. This is an open-access article distributed under the terms of the Creative Commons Attribution License (CC BY). The use, distribution or reproduction in other forums is permitted, provided the original author(s) and the copyright owner(s) are credited and that the original publication in this journal is cited, in accordance with accepted academic practice. No use, distribution or reproduction is permitted which does not comply with these terms. 